\title{
La Educación por las Nubes: Una experiencia educativa desde el confinamiento
}

\author{
Education through the Clouds: an educational experience in \\ lockdown
}

\author{
Natalia Tello Burgos \\ Universidad de Granada (España) \\ tellonatalia@gmail.com
}

\section{Antonio Llanas Torres}

IES. Sol de Portocarrero. Granada (España)

antoniollanas@gmail.com

\author{
Recibido 13/09/2020 Revisado 10/10/2020 \\ Aceptado 11/10/2020 Publicado 31/10/2020
}

\section{Resumen:}

El presente estudio expone una experiencia educativa con el alumnado del instituto IES Sol de Portocarrero de la provincia de Almería de la última etapa de la Educación Secundaria Obligatoria (ESO), en la materia de Educación plástica, visual y audiovisual en los meses de cuarentena a causa del COVID-19.

Durante los meses de confinamiento se han llevado a cabo diferentes actividades expresamente diseñadas para esta situación en las que se han trabajado elementos del currículo de secundaria a través de varias herramientas digitales proporcionadas por la compañía Google.

Se han tenido en cuenta cuestiones como la igualdad de oportunidades para que todo el alumnado pueda realizarlas, además de la interacción entre las actividades, la secuencialización entre ellas y la evaluación de estas mediante distintos niveles de ejecución.

Este estudio presenta cada una de las herramientas empleadas, las actividades llevadas a cabo, los resultados obtenidos y la evaluación de los estudiantes acerca de toda esta experiencia educativa.

Sugerencias para citar este artículo,

Tello Burgos, Natalia, Llanas Torres, Antonio (2020). La educación por las nubes: Una experiencia educativa desde el confinamiento. Tercio Creciente (Monográfico extraordinario II), págs. 103-113, https://dx.doi.org/10.17561/rtc.extra2.5742

TELLOS BURGOS, NATALIA, LLANAS TORRES, ANTONIO . La educación por las nubes: Una experiencia educativa desde el confinamiento. Tercio Creciente (Monográfico extraordinario II), octubre 2020. pp. 103-113, https://dx.doi.org/10.17561/tc.extra2.5742 


\section{Abstract:}

This study presents an educational experience with students from the IES Sol de Portocarrero (Almería) in the last stage of the Educación Secundaria Obligatoria (ESO), in the subject of Educación plástica, visual y audiovisual during the months of quarantine due to the COVID-19.

Throughout the months of lockdown, several activities have been carried out specifically designed for this situation, working on elements of the secondary school curriculum, using various digital tools supplied by the company Google.

We have taken into account issues such as equal opportunities so that all students can carry them out, as well as the interaction between the activities, the sequencing between them, and the evaluation of these activities with different levels of execution.

This study presents each of the tools used, the activities carried out, the results obtained, and the evaluation of the students about this whole educational experience.

Palabras Clave: Educación Plástica, Online, Formación, Divulgación, Confinamiento

Key words: Art Education, online, formation, divulgation, confinement.

Sugerencias para citar este artículo,

Tello Burgos, Natalia, Llanas Torres, Antonio (2020). La educación por las nubes: Una experiencia educativa desde el confinamiento. Tercio Creciente (Monográfico extraordinario II), págs. 103-113, https://dx.doi.org/10.17561/rtc.extra2.5742

TELLOS BURGOS, NATALIA, LLANAS TORRES, ANTONIO . La educación por las nubes: Una experiencia educativa desde el confinamiento. Tercio Creciente (Monográfico extraordinario II), octubre 2020. pp. 103-113, https://dx.doi.org/10.17561/tc.extra2.5742 


\section{Introducción}

Este año a causa del COVID-19, en el sistema educativo se han puesto de manifiesto diferentes situaciones que, debido al factor sorpresa y a la improvisación, han actuado en contra.

La falta de planificación, la dificultad para acceder a los medios tecnológicos necesarios para el desempeño tanto del alumnado como de los docentes o la disposición de herramientas de Tecnología de Información y Comunicación (TIC) plenamente funcionales, generales para todos o incluso oficiales, son algunos de los numerosos ejemplos. Por no hablar del desconocimiento generalizado sobre el funcionamiento de estas herramientas o la distribución y organización de los contenidos u horarios de las clases. Pero no todo han sido malas experiencias, carencias o dificultades, esta situación ha actuado también a favor de los procesos de enseñanza y aprendizaje del alumnado.

Uno de los aspectos principales ha sido el contar con la posibilidad de poner en funcionamiento las actitudes digitales que tanto se valoran en la actualidad como son: el conocimiento digital, la adaptabilidad a diferentes situaciones y medios, la responsabilidad individual y colectiva, el aprendizaje contínuo, la empatía o la creatividad... Todas ellas en beneficio de la formación de los estudiantes ya que vivimos en la Era Digital [1], un contexto social cada vez más informatizado y fundamentado en la conectividad.

De esta manera la presente propuesta expone una experiencia educativa con el empleo de diferentes herramientas digitales con las que se trabaja actualmente $4^{\circ}$ de la ESO en la materia de Educación Plástica, Visual y Audiovisual del instituto IES Sol de Portocarrero de la Cañada, en la provincia de Almería. Además, se expondrán los resultados obtenidos y las valoraciones por parte del alumnado que ha participado en las diferentes actividades propuestas.

\section{Herramientas digitales}

Se han empleado diferentes servicios que proporciona la compañía Google. Todos ellos se han complementado de manera que, pese al escaso tiempo que se ha tenido para reaccionar a esta nueva condición educativa centrada en el uso de internet [2], al alumnado de este año se le ha podido ofrecer una docencia online individualizada, formal y completa [3].

También se han tenido en cuenta las características de todo el alumnado que ha asistido a las clases, intentando, en todo momento, romper aquellas barreras y limitaciones que pudieran surgir en la práctica educativa al trabajar en este formato, íntegramente online. 
ISSN: 2340-9096

https://dx.doi.org/10.17561/rtc.extra2.5742

\subsection{Google Classroom}

La herramienta principal que se ha empleado ha sido Google Classroom (en adelante GClassroom) que garantiza una organización de las clases en diferentes secciones y con la que es posible articular las otras herramientas empleadas. La estructura que presenta GClassroom ha permitido, de manera clara e intuitiva gestionar los trabajos del alumnado, sus calificaciones y su actividad en las clases online. GClassroom pone a disposición del alumnado y del docente secciones como:

- El tablón, donde se han colgado noticias o avisos importantes de carácter público y que ha funcionado como el tablón de una red social, y que como docentes hemos podido gestionar la información (mover, borrar y editar los mensajes). Un entorno con el que el alumnado está, en su mayoría, familiarizado por lo que la intervención de estos ha sido fluida y constante.

-El trabajo de clase, para la propuesta de las actividades y donde las tareas programadas se han podido organizar por fechas de entrega (gestionables con Google Calendar). Además, se han podido incluir diferentes recursos como imágenes, enlaces web o material teórico para que el alumnado contase con apoyo visual y documental para las actividades. Esto ha supuesto una ventaja considerable respecto a las clases tradicionales ya que a "un clic" el estudiante ha podido consultar diferentes fuentes, todas ellas seleccionadas y revisadas previamente, que han ampliado y completado las explicaciones del docente.

-Las personas, es decir, los participantes, tanto alumnado como docentes que hemos participado en el aula. Con diferentes niveles de acceso y permisos de edición, todos los usuarios de la GClassroom aparecemos en esta sección. Además, clicando en cada uno de los nombres es posible acceder a su información de perfil o al registro de actividad en la clase.

- Las calificaciones en esta sección se incluyen las rúbricas de evaluación, visibles a todo el alumnado, para su consulta y donde se han realizado el seguimiento de las entregas. También ha sido posible calificar las tareas, notificado al alumnado no sólo a través de la GClassroom sino también a través de correo asociado al perfil con el que partipan en la clase.

Es importante resaltar que esta herramienta ha posibilitado la comunicación con el alumnado de manera pública, a través del tablón, y privada mediante mensajes individuales, del mismo modo ha permitido el envío de correos electrónicos. De esta manera la relación con el alumnado se ha mantenido de forma fluida, estableciendo horarios (las horas de las clases presenciales) en los que el docente ha estado conectado y ha podido responder a tiempo real las dudas y cuestiones que han ido surgiendo en el desarrollo de las actividades y clases. Aun así, en algunos casos, ha sido muy complicada mantener la relación con el alumnado debido a varios factores, siendo principalmente los medios tecnológicos disponibles, la coordinación con los horarios de otras materias, la cantidad de trabajos exigidos y el desgaste o cansancio digital [4] con el tiempo. 


\subsection{Google Drive}

Para el almacenamiento de todos los trabajos escritos, exposición de imágenes o presentaciones del alumnado se ha empleado Google Drive (en adelante GDrive). Esta herramienta, junto con su homóloga Dropbox, es ampliamente empleada para alojar archivos en la nube, y así almacenar y sincronizar archivos online y entre ordenadores pudiendo compartir y editar archivos con varias personas o grupos de trabajo y mediante diferentes plataformas. Por lo que ha resultado ideal para alumnos y profesores, ya que desde cualquier dispositivo (ordenadores, móviles o tablets) se ha podido acceder a los archivos.

Además, GDrive ha permitido la corrección simultánea de los textos o trabajos escritos mediante la inclusión sobre el propio texto o la inserción de comentarios, preguntas o asuntos a resolver por el estudiante, que quedan en el margen derecho del texto y resaltados en otro color.

Dentro del panel de trabajo de GDrive ha sido posible gestionar y acceder a todos los trabajos compartidos por cada estudiante, así como a sus calificaciones y rúbrica de evaluación. Además, cuenta con un chat donde hablar de manera privada con el alumnado tanto si se encuentra online como si no, pudiendo leerlos cuando conecte.

\subsection{Google Meet}

Esta herramienta ha supuesto una gran ventaja en la docencia online, ya que la consideramos totalmente necesaria e imprescindible para una docencia como esta, que se ha llevado a cabo, íntegramente en línea.

Google Meet (en adelante GMeet) se ha utilizado para apoyar toda la teoría dada, mediante tutorías grupales que han fomentado la participación del alumnado en las actividades y asegurado el contacto con ellos. Cabe resaltar que en la situación que hemos experimentado ha resultado de gran importancia mantener una relación lo más cercana posible que, aunque haya sido a través de pantallas, es de vital importancia tanto para la relación alumno-docente como para alimentar la relación entre ellos [5].

Gracias a estas sesiones en grupo, el alumnado ha podido plantear sus dudas en directo y resolverlas sobre la marcha, sin tener que esperar a que el profesor conecte, que vea el mensaje y que conteste. Esto ha facilitado la comprensión de las actividades y de la teoría, ha agilizado las clases y ha supuesto una gran diferencia en la gestión de la asignatura en este periodo.

\subsection{Google Sites}

En la docencia online impartida se ha empleado Google Sites (en adelante GSites) para la difusión de los trabajos del alumnado y también para hacer visible el desarrollo de enseñanza y aprendizaje durante la cuarentena. 
Revista de Estudios en Sociedad, Artes y Gestión Cultural

El ejercicio en las aulas rara vez, sale de las clases o del centro y se expone o es admirado por el resto de la comunidad educativa. Por eso se ha creado esta web Art\&Student ${ }^{1}$ dándole difusión a través del blog institucional del centro (Ilustración 1). Todo este trabajo se ha realizado debido a que consideramos que la educación artística posibilita, de manera natural, la puesta en valor del esfuerzo de los estudiantes, por lo que es muy interesante aprovechar esta facultad natural del área para dar salida a estos trabajos y así fomentar la competitividad creativa y, sobre todo, la conexión entre la materia impartida en clase con una de sus funciones en el mundo real: las exposiciones de arte y la promoción de la cultura visual.

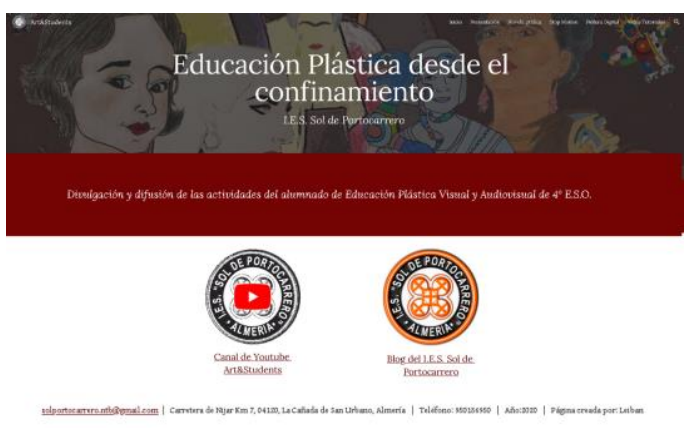

Ilustración 1: Página principal de la web Art\&Student, creada para el instituto IES Sol de Portocarrero (Almería)/Diseño web por Leiban/ Google Sites.

\subsection{YouTube}

La última herramienta empleada ha sido la plataforma YouTube, cada vez más utilizada por docentes como apoyo educativo [6 y 7].

Para este particular se ha creado el canal del profesor $\underline{\text { Natt\&Art }}^{2}$ donde se han colgado tutoriales y píldoras de conocimiento que han ayudado al alumnado a aclarar dudas sobre las actividades y, para los que no han podido asistir a las reuniones en GMeet, a realizar las tareas. La ventaja de tener este material en YouTube o en cualquier otro repositorio, es que ofrece la oportunidad de ver varias veces las clases. Además, esta plataforma en concreto, permite el etiquetado (palabras clave) en la descripción de los vídeos por lo que la propia web es capaz de sugerir, si las etiquetas son correctas, contenido adicional que puede complementar la formación a los alumnos con más inquietudes.

Para la puesta en valor del desempeño del alumnado y para aumentar su motivación y participación en las actividades, se ha creado otro canal, ${\operatorname{Art} \& S t u d e n t^{3}}^{3}$, el cual está dirigido por el departamento de dibujo del centro I.E.S. Sol de Portocarrero y que muestra los trabajos del alumnado. El empleo de esta plataforma, tan utilizada por los adolescentes para comunicarse y

\footnotetext{
${ }^{1}$ Enlace web diponible en esta URL https://sites.google.com/view/artstudent

${ }^{2}$ Enlace web diponible en esta URL https://www.youtube.com/channel/UCDBbkxmvylgNuT5boxGjbrw

${ }^{3}$ Enlace web disponible en esta URL https://www.youtube.com/channel/UCOJmzPSOPbt8uG993CG3ykQ
} 
como método de interacción audiovisual [8 y 9], logra un uso de las TIC centrado en la educación, potenciando el uso responsable y adecuado de internet con contenidos adaptados y libres de derechos. De esta manera su empleo contribuye a la enseñanza y aprendizaje del alumnado, aportando criterio y consciencia sobre el consumo de información [10].

\section{Actividades propuestas}

Debido a la situación derivada del estado de alarma por el COVID-19, para la etapa final del curso escolar 2019/2020 se han propuesto una serie de actividades diseñadas específicamente para su desarrollo online. Estas se han clasificado en dos grupos según la competencia a trabajar:

-Investigación y reflexión, donde el alumnado ha necesitado de su capacidad crítica e investigativa, empleando como motor su propio deseo y curiosidad por el tema planteado.

-Habilidad digital, donde se ha puesto a prueba la capacidad del alumnado al emplear sus actitudes y aptitudes digitales a favor de su propia educación y adaptadas al currículo de secundaria.

Todos los trabajos del alumnado que ha participado en las actividades y firmado el consentimiento de difusión, pueden verse en la web Art\&Student.

\subsection{Investigación y reflexión}

Dentro del primer grupo de actividades se crearon dos tareas con las que el alumnado ha trabajado diferentes competencias enmarcadas en la etapa de secundaria y en el área de las artes plásticas.

La primera actividad consistió en la elaboración de un comentario estético, donde el alumnado tuvo que realizar varias tareas. Por un lado, investigar y navegar por internet en busca de una obra considerada por el público en general o por el mismo, como "Obra de arte". Además de seleccionar una de ellas en base a su propio juicio y finalmente, escribir un breve texto con su valoración personal acerca de la obra elegida. Con esta actividad el alumnado puso en marcha su capacidad de introspección y reflexión más personal, permitiéndonos valorar como docentes su competencia lingüística escrita (vocabulario específico, sentimiento estético...), por medio de la presentación de un archivo a través de GDrive, y oral (capacidad comunicativa, expresión corporal, dicción...) mediante la exposición y explicación de su visión de la obra a sus compañeros mediante GMeet.

La segunda actividad fue la creación de una novela gráfica o historia novelada, poniendo en valor la obra de artistas mujeres de las vanguardias. Para ello se les facilitó una lista abierta de artistas de las que cada alumno tuvo que elegir libremente una, investigar sobre su vida y obra y con esa información elaborar una novela con los materiales que desease. Además, se les facilitaron diferentes recursos como ejemplos online de novelas gráficas, material del tipo 
"tutorial" realizado por el propio docente y más información para que pudieran inspirarse y trabajar cómodamente.

La presentación de estos trabajos se hizo por medio de diferentes formatos, quedando abierto a la elección de los estudiantes. Se presentaron a través de medios audiovisuales (subidos al canal de YouTube: Art\&Students) y mediante imágenes o en presentaciones subidas a GDrive.

\subsection{Habilidad digital}

Formando el segundo grupo de actividades el alumnado tuvo que realizar un trabajo audiovisual en stop motion y una portada para el vídeo mediante pintura digital. El tema sugerido para la realización de ambas actividades fue sobre la actualidad 2020, es decir, su vida, sus rutinas y pensamientos durante el periodo de confinamiento. De esta forma, se ha conseguido dotar a la actividad de un carácter novedoso donde expresar de manera creativa con los materiales encontrados por casa, sus emociones y preocupaciones.

Para su desarrollo se les ofreció un tutorial sobre el uso de dos apps de móvil o tablet, totalmente gratuitas fomentando el empleo de las nuevas tecnologías, su utilidad para el desempeño de tareas artísticas y su proyección a la realidad que nos rodea. Además, el trabajo en este tipo de actividad propició un aprendizaje sobre el tema de los derechos de autor y las posibilidades de acceso e intercambio de las licencias Creative Commons.

De esta manera los estudiantes tuvieron la ocasión de trabajar con conceptos y herramientas actuales, que por lo general están presentes en su día a día y que sin ser conscientes utilizan. Además de buscar soluciones creativas para el desarrollo de stop motion, improvisando o reciclando materiales y todo ello asociado al currículo previsto para su formación.

\section{Resultados y evaluación}

En todo sistema educativo es importante disponer de un feedback de los docentes involucrados en el proceso de enseñanza y aprendizaje a modo de memoria, pero más aun de los estudiantes. Por esta razón, hemos considerado de gran importancia la opinión del propio alumnado sobre su formación como sujeto activo y actor principal en el proceso educativo. Ya que su valoración nos ayuda a crecer, detectando los puntos fuertes y débiles en nuestra metodología docente.

En este apartado se van a comentar las principales consideraciones por parte del alumnado participante sobre las actividades realizadas, el formato íntegramente online de la docencia y sobre su desempeño y el del propio docente. 
Se ha realizado una encuesta a través de Google Forms, donde de manera obligatoria han participado todos los alumnos que han realizado las actividades programadas, la participación del alumnado en las tareas durante el confinamiento ha sido de un $64 \%$ disminuyendo su asistencia a las GMeet y las entregas a final de curso, por lo tanto, hemos contado para la evaluación de la experiencia educativa con un total de 32 opiniones.

Los motivos de la participación en las clases han sido variados, pero es principalmente, la escasa valoración, por parte de la comunidad educativa, de la materia de artes plásticas lo que ha contribuido al abandono paulatino de la asignatura. Esto, junto a la no consideración, en la calificación final, del desempeño durante el confinamiento, han sido los dos factores principales. Aun así, la percepción general es positiva ya que el alumnado participante se ha sentido muy motivado y a gusto con la metodología y las actividades desarrolladas.

Con respecto a la valoración de las actividades propuestas, la que mejor acogida ha tenido por parte del alumnado ha sido el trabajo audiovisual de stop motion. El alumnado ha considerado esta actividad muy creativa, motivadora y sobre todo diferente en comparación con lo que suelen hacer. Frente a la peor valorada, el comentario estético, la cual han encontrado bastante aburrida.

Sobre las herramientas y apps empleadas en clase, todos los estudiantes han estado de acuerdo con que deberían implementarse en futuros años y con que las habilidades y herramientas digitales empleadas son muy útiles en su entorno, debido a que muchos de ellos las utilizan motu proprio en su día a día. Además, el $73 \%$ del alumnado encuestado considera que las herramientas de difusión (la web de GSites y el canal de YouTube) han propiciado su participación y un mayor esfuerzo y consideración por realizar mejor las actividades.

Continuando con la valoración de la metodología online, la mayoría de los estudiantes (64\%) opina que trabajar con herramientas TIC es un sistema compatible con las clases presenciales, aunque un buen porcentaje (32\%) prefiere que predominen las clases en el aula. El resto no se ha sentido cómodo con el sistema online.

Con respecto a los pros y contras de este sistema docente, en general se considera que lo más positivo ha sido la flexibilidad en las fechas de entrega de las actividades y el acceso a internet para trabajar. El aspecto peor valorado ha sido la carga de trabajo y el hecho de tener que resolver dudas y problemas que surgen al realizar las actividades sin tener al profesor o a los compañeros cerca. En esta línea, se les preguntó por el factor que más echaron de menos durante el periodo de clases en cuarentena y la respuesta más repetida (92\% de las valoraciones) ha sido, los compañeros de clase.

Finalmente, se les preguntó sobre su propio desempeño en las actividades y todos están muy satisfechos con su trabajo, considerando en su mayoría que la motivación y el tipo de actividades propuestas han sido la razón principal. Asimismo, todo el alumnado ha evaluado muy positivamente la actuación del profesorado del departamento de dibujo, resaltando el trato adecuado, el tiempo de respuesta y el tipo de metodología utilizada para impartir las clases. 
En general, podemos afirmar que la evaluación ha sido bastante positiva, el alumnado se ha sentido satisfecho con el tipo de clases impartidas durante el periodo de confinamiento y con su propio trabajo. Es importante que los estudiantes no se hayan sentidos frustrados ni abandonados durante una docencia complicada para todos. Gracias a esta experiencia se ha podido apreciar el funcionamiento del alumnado cuando dependen de sí mismos para afrontar los retos que supone su educación y su, más que aceptable, adaptación a la situación y a la metodología implementada.

\section{Conclusiones}

Debido a la situación que hemos vivido estos últimos meses se nos ha presentado la posibilidad de poner en funcionamiento herramientas de las que ya disponíamos pero que no se venían utilizando de manera sistemática o eficiente.

Esta experiencia demuestra que es posible una hibridación de la educación presencial y online, empleando para ello diferentes plataformas, como es el caso que nos ocupa, que complementen la formación de manera significativa para el estudiante.

También se ha puesto de manifiesto el problema que supone una docencia íntegramente online ya que por lo menos en la materia de Educación plástica, visual y audiovisual, y sobre todo en la etapa de la ESO, es imprescindible el trato directo con el alumnado y ellos necesitan del docente y de sus compañeros para desarrollarse correctamente como individuos completos y equilibrados.

\section{Referencias}

[1] Viñals Blanco, Ana; Cuenca Amigo, Jaime (2016) "El rol del docente en la era digital". Revista interuniversitaria de Formación del Profesorado 30 (2). Universidad de Zaragoza. Recuperado de https://www.redalyc.org/jatsRepo/274/27447325008/html/index.html

[2] Villota García, Stefanny Carolina; Zamora López, Gabriela Geraldine; Llanga Vargas, Edgar Francisco (2019) "Uso de Internet como base para el aprendizaje". Revista Atlante: Cuadernos de Educación y Desarrollo. ISSN 1989-4155. Recuperado de https://www.eumed.net/rev/atlante/2019/05/internet-aprendizaje.html

[3] Cabero Almenara, Julio; Córdoba Pérez, Margarita (2009) “Inclusión educativa: inclusión digital”. Revista Educación Inclusiva 2 (1). ISSN: 1130-0876. Recuperado de: https://revistaeducacioninclusiva.es/index.php/REI/article/view/27 
[4] Varsavsky, Julián (2020) “El viaje a la sociedad del cansancio digital”. [Ensayo]. Recuperado de http://repositorio.uasb.edu.ec/bitstream/10644/7153/1/03-ENVarsavsky.pdf

[5] D'Aloisio, Florencia (2014) "La secundaria como espacio-tiempo de sociabilidad: Aprendizajes para alivianar dificultades de vida y afrontar riesgos". Cuadernos de Educación Año XII (12) ISSN 2344-9152. Recuperado de https://revistas.psi.unc.edu.ar/index.php/Cuadernos/article/view/9223

[6] Canal de Youtube: unicoos https://www.youtube.com/user/davidepv

[7] Canal de YouTUbe: unProfesor https://www.youtube.com/user/unprofesorcom

[8] García Jiménez, Antonio; Catalina García, Beatriz y Cruz López de Ayala, María (2016) "Adolescents and YouTube: Creation, Participaction and Consumption". Prisma Social, número especial 1 ISSN 1989-3469. Recuperado de https://dialnet.unirioja.es/servlet/articulo?codigo $=5602003$

[9] Pérez-Torres, Vanesa; Pastor-Ruiz, Yolanda; Abarrou-Ben-Boubaker, Sara (2018) "Los youtubers y la construcción de la identidad adolescente". Recuperado de http://hdl.handle.net/10115/15249

[10] Borba, Domingo y Avalos, Mariano (2019) "El uso responsable y seguro de internet. Aportes para la conformación de la ciudadanía digital”. Argentina. ISBN: 978-987-443487-6. 
Revista de Estudios en Sociedad Artes y Gestión Cultural

ISSN: 2340-9096

https://dx.doi.org/10.17561/rtc.extra2.5742
Monográfico

114

xtraordinario II

Octubre 2020

Investigación 